\title{
Similar Performance of Trabectome and Ahmed Glaucoma Devices in a Propensity Score Matched Comparison
}

Tarek Shazly ${ }^{1}$, Susannah Waxman ${ }^{1}$, Sushma Kola ${ }^{1}$, Kevin B. Kaplowitz², Eric N. Brown ${ }^{3}$, Nils A. Loewen ${ }^{1 *}$

1. Department of Ophthalmology, University of Pittsburgh, Pittsburgh, PA

2. Department of Ophthalmology, VA Loma Linda, Loma Linda University, Loma Linda, CA

3. Department of Ophthalmology, Vanderbilt University Medical Center, Nashville, TN

*corresponding Author:

Nils A. Loewen, MD, PhD

University of Pittsburgh, School of Medicine

Department of Ophthalmology

203 Lothrop St

Pittsburgh, PA 15213

Email: loewen.nils@gmail.com

Phone: 412-944-2554 Fax: 412-647-5119 


\section{Abstract}

Purpose: To apply propensity score matching to Ahmed glaucoma drainage implants (AGI) to trabectome-mediated ab interno trabeculectomy (AIT). Recent data suggests that AIT can produce results similar to AGI which is traditionally reserved for more severe glaucoma.

Methods: AGI and AIT patients with at least one year of follow-up were included. The primary outcome measures were intraocular pressure (IOP), glaucoma medications, and a Glaucoma Index (GI) score. GI reflected glaucoma severity based on visual field, the number of preoperative medications, and preoperative IOP. Score matching used a genetic algorithm consisting of age, gender, type of glaucoma, concurrent phacoemulsification, baseline number of medications, and baseline IOP. Patients without a close match were excluded.

Results: Of 152 patients, 34 AIT patients were matched to 32 AGI patients. Baseline characteristics including ethnicity, IOP, the number of medications, glaucoma type, the degree of VF loss and GI were not significantly different between AIT and AGI. AIT had a preoperative IOP of $23.6 \pm 8.1 \mathrm{mmHg}$ compared to $26.5+10.6$ for $\mathrm{AGI}$. At 12 months, the mean IOP was $15.0 \pm 9 \mathrm{mmHg}$ for AIT versus $15.0 \pm 4 \mathrm{mmHg}$ for AGI $(p=0.8)$, while the number of drops was $2.3 \pm 2.2$ for AIT versus $3.6 \pm 1.3$ for AGI $(p=0.016)$. Only 6 AIT patients (17.6\%) required further surgery within the first 12 months versus 9 (28\%) for AGI. Success, defined as IOP $<21 \mathrm{mmHg},<20 \%$ reduction and no reoperation, was achieved in $76 \%$ of AIT versus $69 \%$ of AGI ( $p=0.48)$. Complications occurred in $13 \%$ of AGI and $0.8 \%$ of AIT.

Conclusions: A propensity score matched comparison of AIT and AGI demonstrated an equivalent IOP reduction through one year. Surprisingly, the AGI group required more glaucoma medications than the AIT group at 6 and 12 months. 


\section{Introduction}

Epibulbar glaucoma drainage devices are used to bypass the abnormal resistance to outflow in moderate to severe glaucoma [1]. Similar to the Krupin glaucoma drainage implant [2], the first valved glaucoma device, the Ahmed glaucoma implant (AGI, New World Medical, Rancho Cucamonga, CA, USA) has a pressure-sensitive valve that is designed to open when the IOP is $8 \mathrm{mmHg}$ to avoid complications related to hypotony during the early postoperative period [3]. In contrast, the Trabectome (Neomedix Inc, Tustin, CA, USA), generates plasma to ablate the trabecular meshwork [4], which constitutes the primary resistance to outflow. This procedure is called ab interno trabeculectomy (AIT). Outflow resistance downstream of the trabecular meshwork and the episcleral venous pressure limit IOP reductions as compared to AGI while also preventing hypotony [5]. Complications are relatively rare and similar to that seen in cataract surgery [6]. AIT also lowers IOP rapidly but has been used only in mild to moderate glaucoma until it was recently described as an option for failed trabeculectomy [7] or failed epibulbar glaucoma drainage implants [8]. AGI and AIT differ considerably in procedure times, cost $[9,10]$, and complication profiles $[6,11]$.

Prior studies of AIT [12-17] and AGI [11,18] suggested a similar postoperative average IOP, but the patient populations might have different in glaucoma severity and type. Recent studies that stratified AIT outcomes by glaucoma severity indicated that patients with a higher preoperative IOP and more severe glaucoma experienced a larger IOP reduction [19-21]. Because of this, we expanded our surgical indications to include those traditionally reserved for AIG and compared the outcomes in the present study. We hypothesized that AIG and AIT achieve a similar pressure reduction when similar glaucoma stages are compared.

Although a randomized controlled trial remains the most effective tool to reduce bias and patient selection, modern statistical matching strategies [22-25] obtain a highly-balanced comparison of 
already existing patient data. They also avoid non-routine practice patterns and a potential bias that may occur during patient recruitment in trials. We have used Coarsened Exact Matching before $[26,27]$ to achieve a balanced comparison, but the challenge with potentially more uneven groups is that only a few patients in each group can be matched precisely. The risk of achieving an overlap of matched features by those means is that regression toward the mean occurs which can make the comparison group look better or worse than they are. In contrast, the method we use here, Propensity Score Matching, uses a predicted probability of group membership to achieve a match $[24,25,28]$.

\section{Methods}

This retrospective chart review was approved by the Institutional Review Board of the University of Pittsburgh (PRO14100026) and adhered to the tenets of the Declaration of Helsinki and the Health Insurance Portability and Accountability Act of 1996. Charts from October 2012 through February 2015 were included that had a follow-up of at least one year. Inclusion criteria were at least 18 years of age undergoing Trabectome-mediated AIT or AGI surgery with at least 12 months of follow-up. All surgeries were performed by the same group of surgeons using the same surgical technique. Neovascular glaucoma was excluded.

The main outcome measure was the postoperative IOP at one year. Secondary measures included the reduction of medications as well as success rates. Failure was defined (after the TVT)[29] as anyone of postoperative IOP $<6$, IOP $>21 \mathrm{mmHg}$, or $<=20 \%$ reduction in IOP from baseline at two consecutive visits after 3 months, or reoperation.

All patients underwent a complete ophthalmologic examination before surgery and at each postoperative visit; this included best-corrected visual acuity, slit-lamp biomicroscopy, Goldmann 
applanation tonometry and funduscopic evaluation. The indication for surgery (including the decision of which surgery to proceed with) was determined by the individual surgeon when the IOP was above the targeted IOP on maximally tolerated medical treatment. Procedures were performed by the same group of surgeons on comparable patient populations. The decisions to resume medications or to reoperate were also made by the individual surgeon.

The glaucoma index (GI) is an indicator of the severity of glaucoma and its relative resistance to treatment [19-21]. It is based on the visual field damage, the number of preoperative glaucoma medications, and the preoperative IOP. Visual field was separated into three categories: mild, moderate, or advanced visual field damage, which was assigned 1, 2, or 3 points, respectively. Preoperative number of medications were divided into four categories: $0-1,2,3$ or 4+, and assigned a value of 1 to 4 , respectively. Baseline IOP was divided into three categories: $<20 \mathrm{mmHg}, 20-29 \mathrm{mmHg}, 30-39 \mathrm{mmHg}$, and greater than $40 \mathrm{mmHg}$ and assigned from 1 to 4 points, respectively. GI was defined as the product of preoperative IOP $\times$ preoperative number of medications $\times$ VF and separated into four groups: $<6$ (Group 1), 6-12 (Group 2), >12-18 (Group 3) and >18 (Group 4).

\section{Statistics}

Cases were matched with propensity-score matching (package “Matching" in R) [30,31] using a genetic algorithm based on age, gender, type of glaucoma, concurrent phacoemulsification, and baseline IOP and medications. Cases of AIT or AGI too different from their counterparts were excluded. Weighted linear regression was significant when $\mathrm{p}<0.05$. A Kaplan-Meier survival curve for right-censored data with $80 \%$ confidence intervals was computed. A Cox proportional hazard model was developed to estimate the effects of 16, 21 and $26 \mathrm{mmHg}$ preoperative IOP on surgical success. Data available beyond the 12 months was included in a secondary analysis and shown in average IOPs, medications, survival 
and hazard graphs.

\section{Surgical Technique}

AIT was performed by creating a $1.8 \mathrm{~mm}$ uniplanar temporal clear corneal incision and ablating approximately 140 to 160 degrees of nasal TM under direct gonioscopic view. For AIT combined with phacoemulsification, the main incision was enlarged to $2.2 \mathrm{~mm}$ followed by a standard phacoemulsification.

AGI tubes (model FP-7) were primed and sutured to the superotemporal sclera $10 \mathrm{~mm}$ posterior to the surgical limbus. The tube was trimmed so that it extended 1-2 mm onto the anterior surface of the iris and covered with a scleral patch graft.

Patients in both groups used topical moxifloxacin and prednisolone acetate $1 \%$ four times per day. AIT patients also received pilocarpine $1 \%$ four times daily for one month then three times daily for one month. All preoperative glaucoma medications were stopped on the day of surgery and were added back later at the discretion of the surgeon. Postoperative clinic visits occurred at day 1, week 1, month 1 , month 3, month 6 and month 12.

\section{Results}

Out of 102 AIT and 49 AGI patients, 66 were included in the study, consisting of 34 individuals in the AIT group and 32 in the AGI group (Fig 1). Using case matching, there was no significant difference in ethnicity, IOP, number of IOP-lowering medications, glaucoma type, the degree of VF loss and GI between AIT and AGI ( $p>0.05)$. Table 1 shows baseline characteristics of each group.

Fig 1. Ahmed glaucoma drainage device and Trabectome to scale. Devices are shown at the correct relative size and in the same eye for easier comparison. The Ahmed glaucoma drainage device has a valve to prevent overfiltration and a silicone plate with a surface area of $184 \mathrm{~mm}^{2}$ (left). The trabectome 
is most commonly inserted through a temporal incision (nasal incision depicted in this illustration), and the trabecular meshwork is ablated along the nasal angle. The tip of the trabectome has a protective footplate that feeds the trabecular meshwork towards the active and passive electrode where plasma is generated (top right). The molecularized tissue is displaced by fluid from the irrigation ports and aspirated at the tip.

Table 1. Baseline data of matched patients.

\begin{tabular}{|c|c|c|c|}
\hline & AIT & AGI & $\mathrm{p}$ \\
\hline $\mathrm{n}$ & 34 & 32 & \\
\hline Age (years) & 69.1 & 67.8 & 0.71 \\
\hline Female (\%) & $61.8 \%$ & $53.1 \%$ & 0.48 \\
\hline Ethnicity (\%) & & & 0.52 \\
\hline Asian & $8.82 \%$ & $3.12 \%$ & \\
\hline Black & $26.5 \%$ & $31.2 \%$ & \\
\hline White & $64.7 \%$ & $65.6 \%$ & \\
\hline Baseline IOP (mmHg) & $23.6 \pm 8.1$ & $26.5+10.6$ & 0.25 \\
\hline Medications (n) & 2.91 & 2.81 & 0.79 \\
\hline With Phaco (\%) & $35.3 \%$ & $31.2 \%$ & 0.58 \\
\hline Glaucoma Type (\%) & & & 0.46 \\
\hline POAG & $52.9 \%$ & $43.8 \%$ & \\
\hline Inflammatory & $5.88 \%$ & $12.5 \%$ & \\
\hline CACG & $8.82 \%$ & $12.5 \%$ & \\
\hline Steroid-induced & $8.82 \%$ & $9.38 \%$ & \\
\hline Other & $23.5 \%$ & $21.9 \%$ & \\
\hline \multicolumn{4}{|l|}{ Degree of VF Loss } \\
\hline Average & $2.4(1-3)$ & $2.5(1-3)$ & 0.5 \\
\hline Mild (1) & $7(20.6 \%)$ & $5(15.6 \%)$ & \\
\hline Moderate (2) & $8(23.5 \%)$ & $5(15.6 \%)$ & \\
\hline Severe (3) & 19 (55.9\%) & $22(68.8)$ & \\
\hline
\end{tabular}




\begin{tabular}{llll}
\hline Glaucoma Index $(\mathrm{GI})$ & & & \\
\hline Average & $2.6(1-4)$ & $2.8(1-4)$ & 0.3 \\
\hline Group 1 & $9(26.5 \%)$ & $5(15.6 \%)$ & \\
\hline Group 2 & $8(23.5 \%)$ & $10(31.3 \%)$ & \\
\hline Group 3 & $6(17.6 \%)$ & $6(18.8 \%)$ & \\
\hline Group 4 & $11(23.4 \%)$ & $11(34.4 \%)$ \\
\hline
\end{tabular}

IOP: intraocular pressure; POAG: primary open angle glaucoma; phaco: phacoemulsification; CACG: chronic angle closure glaucoma; VF: visual field.

AIT group had a preoperative IOP of $23.6 \pm 8.1 \mathrm{mmHg}$ compared to $26.5+10.6 \mathrm{mmHg}$ in $\mathrm{AGI}$. The mean IOP at 1 week was $15.6 \pm 9.8 \mathrm{mmHg}$ for AIT versus $15.7 \pm 7.2 \mathrm{mmHg}$ for AGI $(p=0.9), 15.6 \pm 4.5 \mathrm{mmHg}$ for AIT versus $16.4 \pm 6.4 \mathrm{mmHg}$ for $\mathrm{AGI}(p=0.4)$ at 3 months. At 6 months, the mean IOP was $13.4 \pm 6.2$ $\mathrm{mmHg}$ for AIT versus $16.3 \pm 5.2 \mathrm{mmHg}$ for $\mathrm{AGI}(\mathrm{p}=0.06)$. At 12 months, the mean IOP was $14.5 \pm 8.7 \mathrm{mmHg}$ for AIT versus $14.9 \pm 3.6 \mathrm{mmHg}$ for $\mathrm{AGI}(\mathrm{p}=0.8)$. Fig 2 illustrates the postoperative IOP changes.

Fig 2. Intraocular pressure after Trabectome-mediated ab interno trabeculectomy (AIT) and Ahmed glaucoma implant (AGI). The intraocular pressure (IOP, average \pm standard deviation) started diverging after three months but were not statistically different. Data for 24 months (AIT: $n=24, A G I: n=20$ ) and 30 months ( $A I T: n=18, A G I: n=12$ ) are also shown but were not part of the match due to reduced numbers.

The number of IOP-lowering medications (Fig 3) at three months was $2.7 \pm 1.4$ for AIT versus 3.6 $\pm 1.7(p=0.02)$. At six months, it was $2.6 \pm 1.8$ for AIT versus $3.7 \pm 1.3$ for $A G I(p=0.018)$. At one year, the number of medications was $2.3 \pm 2.2$ for AIT versus $3.6 \pm 1.3$ for $A G I(p=0.016)$.

Fig 3. Glaucoma medications after Trabectome-mediated ab interno trabeculectomy (AIT) and Ahmed 
glaucoma implant (AGI). The number of glaucoma medications (average \pm standard deviation) started diverging after postoperative day 1 through 3 months but was not statistically different. Data for 24 months (AIT: $n=24, A G I: n=20$ ) and 30 months (AIT: $n=18, A G I: n=12$ ) are also shown but were not part of the match due to reduced numbers.

The survival analysis (Fig 4) indicated a similar survival for both AIT and AGI. While the survival declined faster for AIT at 300 days, the difference was not significant (as also evidenced by the confidence intervals that overlap the mean). Only six AIT patients (17.6\%) required further surgery within the first 12 months $204+136$ days compared to nine (28\%) AGI patients who needed additional procedures after an average of $186+114$ days from the time of their AGI surgery. The six AIT patients underwent two AGI surgeries and four transscleral diode cyclophotocoagulation (CPC) procedures to control IOP better. The nine additional procedures in the AGI group included three AGI revisions, four Baerveldt glaucoma shunt implantations, and two CPCs. Success was achieved in $76 \%$ of AIT and $69 \%$ of AGI $(p=0.48)$.

Fig 4. Survival analysis. Kaplan-Meier survival curve with $80 \%$ confidence intervals. AIT: Trabectome-mediated ab interno trabeculectomy. AGI: Ahmed glaucoma drainage implant.

Cox proportional hazard modeling (Fig 5) was only possible for the preoperative IOP due to the limited number of eyes available for potential covariates. The Cox hazard model for different levels of preoperative IOP suggested that AIT did slightly better, lagging approximately 250 to 500 days behind AGI before survival had decreased similarly. However, due to the significant overlap of the confidence intervals, this was not statistically significant. Survival was best for higher preoperative IOPs with both 
surgical modalities.

Fig 5. Cox hazard model with different IOP criteria. The bands are the $80 \%$ confidence intervals. AGI: Ahmed glaucoma drainage implant. AIT: Trabectome-mediated ab interno trabeculectomy.

\section{Complications}

After AIT, the only complication was cystoid macular edema (CME) that occurred in a single case (0.8\%) which was combined with phacoemulsification. After AGI, there were choroidal effusions in $7 \%$, hypotony past three months in $2 \%$, and diplopia in $2 \%$. Visual acuity decreased by $\geq 2$ Snellen lines after one year in $0 \%$ of AIT and $3 \%$ of AGI.

\section{Discussion}

In this study, we found that Trabectome-mediated ab interno trabeculectomy (AIT) and Ahmed glaucoma drainage devices (AGI) achieved a similar IOP reduction but have a complication profile that favors AIT. Although AIT is a mature microincisional glaucoma surgery that was introduced almost 15 years ago, no study has compared trabecular bypass or ablation to traditional glaucoma surgeries. We used propensity score matching to compare AIT, which enhances outflow along the physiological, conventional outflow route and Ahmed glaucoma implants, a valved epibulbar drainage device, which allows bypassing both the conventional and the uveoscleral outflow. Because these surgeries involve such fundamentally different mechanisms and surgical effort, AGIs are typically used in advanced glaucomas with a high IOP while AIT is commonly performed in mild glaucomas with straightforward access to the trabecular meshwork. We expanded our practice pattern to use AIT as a first surgery for 
even uncontrolled glaucomas often reserved for AGI because our studies suggested that AIT can be used after failed trabeculectomy [7], in narrow angles [32] and more severe glaucoma [19-21]

The AGI group required a reintervention rate that was 1.6 times more frequent than AIT while also requiring more medications. In contrast, patients who underwent AIT had no serious complications. AIT offered a safer alternative to a mean final IOP of $14.5 \pm 8.7 \mathrm{mmHg}$, a $30 \%$ decrease from baseline. The final IOP achieved here in AIT and reduction of medications is quite similar to the mean of the largest AIT series ( $n=4,659)$, in which the mean IOP fell from 23.1 to 16.2 after 12 months ( $30 \%$ decrease) on 0.7 fewer medications [33]. Analysis of both the degree of VF loss as well as the GI revealed no statistical difference in glaucoma severity between the two groups. The additional data presented here beyond the focus of 1-year outcomes suggests that both procedures remain comparable for approximately 2.5 years.

Survival analysis is helpful to detail how much IOP data is being censored, for instance when IOP data is no longer included in the mean IOP because of a second surgery. In one study on AIT ( $n=246)$, the mean final IOP after four years was $12 \mathrm{mmHg}$ (45\% decrease from baseline) on 0.5 fewer medications, yet paradoxically, the authors recommended that trabectome should only be used when the goal IOP is $>21 \mathrm{mmHg}[15,34]$. The main reason was the guarded success rates because only $64 \%$ of cases met TVT success criteria [29] after one year and only $28 \%$ were successful using an IOP cutoff of $18 \mathrm{mmHg}$. In contrast, our study showed no statistically significant difference between the success rates for both groups. Compared to our success rate, the $A B C$ trial reported a higher success rate of $84 \%$ for $A G I$ at one year. As a broader measure of failure, the reoperation rate was 1.6 times greater in AIG than in AIT. Likely the main reason why AIT has never been reported to be more successful than penetrating surgery is that both trabeculectomy and aqueous shunts bypass not only the site of highest resistance to aqueous outflow in the TM, but also the proximal collector channel pathway that limits IOP to 
approximately twice of that of the episcleral venous pressure. This indicates that a yet to be identified outflow resistance must exist. We applied a Cox hazard model to estimate the effects of covariates. At least 10 to 15 observations are needed for each parameter fit in a regression model to prevent overfitting [35]. Given the approximately 60 eyes that were in this dataset after matching, this directed us to attempt to fit no more than four to six covariates, but because of the number of failures in the survival analysis, only two to three covariates could be attempted to fit. We assumed a priori that baseline IOP would have an effect on the outcome of surgery because at a minimum the IOP threshold for failure depended on the preoperative IOP. Therefore, a Cox proportional hazards model should at least model a linear relationship between baseline IOP and subsequent surgical failure while additional degrees of freedom would be desirable. Our prior studies indicated that the effect of prior cataract surgery is extremely limited or absent $[26,27]$ even in narrow angles [32]. Because of the limited number of eyes in this analysis, we only modeled IOP.

It is surprising that AGIs that bypass the conventional outflow tract are not more successful than AIT that relies on patency of a functioning outflow system that is downstream of the ablated trabecular meshwork. Recent discoveries of segmental [36,37], circumferential flow [38-40], and fascinating discoveries of valve like elements in collector channels $[4,41]$ highlight the complexity of the conventional outflow route. The advantage of tube shunts to bypass those is likely negated by the fibrosis and chronic foreign body reaction to a silicone drainage implant that is patient specific and cause a variable diffusion barrier [42].

Because a conjunctival bleb and an implant are avoided entirely in AIT, it is considerably safer than either trabeculectomy [43] or aqueous shunts. Complications in the $A B C$ required intervention in $42 \%$ of Baerveldt glaucoma drainage devices and $26 \%$ of AGIs [44]. The rate of endophthalmitis in a large series of 542 aqueous shunts was 1.7\% [45]. Endophthalmitis has only been reported once after AIT [46] 
and may approach the rate of phacoemulsification (i.e. $0.04 \%$ of 1 million cases) [47]. In our study, AIT only had a single complication of CME in one case (0.9\%) compared to choroidal effusions in $7 \%$ of $\mathrm{AGI}$ with $41 \%$ requiring further interventions in the form of cyclodestruction or additional tube shunts. The rate of vision loss is also high: after one year, two or more Snellen lines of visual acuity were lost in 30-34\% for both AGIs and Baerveldt implants in the ABC, AVB, and TVT studies. Most of the AIT literature directly specifies that no patient in the study lost two lines of vision. There is a single study that noted that 13 patients (5\%) lost >2 lines of vision, but there is no clarification given specifying the reason for vision loss or whether those patients had received combined phaco-AIT versus standalone AIT [15]. Persistent hypotony is exceedingly rare [6]. The only patients in our study who lost $>2$ Snellen lines of visual acuity were all in the AGI group (3\%).

Limitations of this study were the modest number of AGI-only patients of a single surgeon (NAL) that resulted in a limited recruitment window for the match. We have since started to combine both AIT and AGI to achieve an even lower IOP on fewer medications in more advanced glaucoma.

\section{Conclusions}

Using propensity score matching, AIT resulted in an IOP reduction, final IOP and success rate at one year that is similar to AGI in groups of equivalent glaucoma severity. Patients who underwent AIT required fewer interventions, fewer medications and had a more favorable complication profile. All of these advantages combined with the fact that the conjunctiva is not violated and that no hardware is implanted make AIT an appropriate first surgical option for patients with medically uncontrolled glaucoma. Results of this study will allow for the design of future comparative studies as well as to justify crossover in the case of failure. 


\section{References}

1. Hong $\mathrm{C}-\mathrm{H}$, Arosemena A, Zurakowski D, Ayyala RS. Glaucoma drainage devices: a systematic literature review and current controversies. Surv Ophthalmol. 2005;50: 48-60.

2. Krupin T, Podos SM, Becker B, Newkirk JB. Valve implants in filtering surgery. Am J Ophthalmol. Elsevier; 1976;81: 232-235.

3. Coleman AL, Hill R, Wilson MR, Choplin N, Kotas-Neumann R, Tam M, et al. Initial clinical experience with the Ahmed Glaucoma Valve implant. Am J Ophthalmol. 1995;120: 23-31.

4. Fallano K, Bussel I, Kagemann L, Lathrop KL, Loewen N. Training strategies and outcomes of $a b$ interno trabeculectomy with the trabectome. F1000Res. 2017;6.

doi:10.12688/f1000research.10236.1

5. Brubaker RF. Goldmann's equation and clinical measures of aqueous dynamics. Exp Eye Res. 2004;78: 633-637.

6. Kaplowitz K, Bussel II, Honkanen R, Schuman JS, Loewen NA. Review and meta-analysis of ab-interno trabeculectomy outcomes. Br J Ophthalmol. 2016;100: 594-600.

7. Bussel II, Kaplowitz K, Schuman JS, Loewen NA, Group TS, Others. Outcomes of ab interno trabeculectomy with the trabectome after failed trabeculectomy. Br J Ophthalmol. BMJ Publishing Group Ltd.; 2014;99: 258-262.

8. Mosaed S, Chak G, Haider A, Lin KY, Minckler DS. Results of Trabectome Surgery Following Failed Glaucoma Tube Shunt Implantation: Cohort Study. Medicine . 2015;94: e1045.

9. Iordanous Y, Kent JS, Hutnik CM, Malvankar-Mehta MS. Projected Cost Comparison of Trabectome, iStent, and Endoscopic Cyclophotocoagulation Versus Glaucoma Medication in the Ontario Health Insurance Plan. J Glaucoma. 2013; doi:10.1097/IJG.0b013e31829d9bc7

10. Kaplan RI, De Moraes CG, Cioffi GA, Al-Aswad LA, Blumberg DM. Comparative Cost-effectiveness of the Baerveldt Implant, Trabeculectomy With Mitomycin, and Medical Treatment. JAMA Ophthalmol. archopht.jamanetwork.com; 2015;133: 560-567.

11. Christakis PG, Kalenak JW, Zurakowski D, Tsai JC, Kammer JA, Harasymowycz PJ, et al. The Ahmed Versus Baerveldt study: one-year treatment outcomes. Ophthalmology. 2011;118: 2180-2189.

12. Ting J, Damji K, Stiles M. Ab interno trabeculectomy: Outcomes in exfoliation versus primary open-angle glaucoma. J Cataract Refract Surg. 2012;38: 315-323.

13. Vold SD. Ab Interno Trabeculotomy With the Trabectome System: What Does the Data Tell us? Int Ophthalmol Clin. 2011;51: 65-81.

14. Jea SY, Francis BA, Vakili G, Filippopoulos T, Rhee DJ. Ab interno trabeculectomy versus trabeculectomy for open-angle glaucoma. Ophthalmology. 2012;119: 36-42.

15. Ahuja Y, Ma Khin Pyi S, Malihi M, Hodge DO, Sit AJ. Clinical results of ab interno trabeculotomy using the trabectome for open-angle glaucoma: the Mayo Clinic series in Rochester, Minnesota. Am 
J Ophthalmol. 2013;156: 927-935.e2.

16. Maeda M, Watanabe M, Ichikawa K. Evaluation of trabectome in open-angle glaucoma. J Glaucoma. 2013;22: 205-208.

17. Francis BA, Minckler D, Dustin L, Kawji S, Yeh J, Sit A, et al. Combined cataract extraction and trabeculotomy by the internal approach for coexisting cataract and open-angle glaucoma: initial results. J Cataract Refract Surg. 2008;34: 1096-1103.

18. Budenz DL, Barton K, Feuer WJ, Schiffman J, Costa VP, Godfrey DG, et al. Treatment outcomes in the Ahmed Baerveldt Comparison Study after 1 year of follow-up. Ophthalmology. 2011;118: 443-452.

19. Loewen RT, Roy P, Parikh HA, Dang Y, Schuman JS, Loewen NA. Impact of a Glaucoma Severity Index on Results of Trabectome Surgery: Larger Pressure Reduction in More Severe Glaucoma. PLoS One. 2016;11: e0151926.

20. Dang Y, Roy P, Bussel II, Loewen RT, Parikh H, Loewen NA. Combined analysis of trabectome and phaco-trabectome outcomes by glaucoma severity. F1000Res. 2016;5: 762.

21. Roy P, Loewen RT, Dang Y, Parikh HA, Bussel II, Loewen NA. Stratification of phaco-trabectome surgery results using a glaucoma severity index. BMJ Ophth in press. 2017; doi:10.13140/RG.2.1.1244.5849

22. lacus SM, King G, Porro G. CEM: Software for coarsened exact matching. J Stat Softw. American Statistical Association; 2009;30: 1-27.

23. lacus SM, King G, Porro G. Causal Inference without Balance Checking: Coarsened Exact Matching. Polit Anal. 2012;20: 1-24.

24. Rubin DB, Thomas N. Combining Propensity Score Matching with Additional Adjustments for Prognostic Covariates. J Am Stat Assoc. 2000;95: 573-585.

25. Seeger JD, Williams PL, Walker AM. An application of propensity score matching using claims data. Pharmacoepidemiol Drug Saf. Wiley Online Library; 2005;14: 465-476.

26. Parikh HA, Bussel II, Schuman JS, Brown EN, Loewen NA. Coarsened Exact Matching of Phaco-Trabectome to Trabectome in Phakic Patients: Lack of Additional Pressure Reduction from Phacoemulsification. PLoS One. 2016;11: e0149384.

27. Neiweem AE, Bussel II, Schuman JS, Brown EN, Loewen NA. Glaucoma Surgery Calculator: Limited Additive Effect of Phacoemulsification on Intraocular Pressure in Ab Interno Trabeculectomy. PLoS One. Public Library of Science; 2016;11: e0153585.

28. Rosenbaum PR, Rubin DB. The Central Role of the Propensity Score in Observational Studies for Causal Effects. Biometrika. [Oxford University Press, Biometrika Trust]; 1983;70: 41-55.

29. Gedde SJ, Schiffman JC, Feuer WJ, Parrish RK 2nd, Heuer DK, Brandt JD, et al. The tube versus trabeculectomy study: design and baseline characteristics of study patients. Am J Ophthalmol. 
2005;140: 275-287.

30. Sekhon JS. Multivariate and propensity score matching software with automated balance optimization: the matching package for R. papers.ssrn.com; 2011; Available:

https://papers.ssrn.com/sol3/papers.cfm?abstract_id=1009044

31. Core Team R. R: A Language and Environment for Statistical Computing [Internet]. Vienna, Austria: R Foundation for Statistical Computing; 2016. Available: http://www.R-project.org/

32. Bussel II, Kaplowitz K, Schuman JS, Loewen NA, Trabectome Study Group. Outcomes of ab interno trabeculectomy with the trabectome by degree of angle opening. Br J Ophthalmol. 2015;99: 914-919.

33. Mosaed S. The first decade of global trabectome outcomes. Clinical \& Surgical Ophthalmology. 2014;32: 1.

34. Minckler D, Mosaed S, Francis B, Loewen N, Weinreb RN. Clinical results of ab interno trabeculotomy using the Trabectome for open-angle glaucoma: the mayo clinic series in Rochester, Minnesota. Am J Ophthalmol. 2014;157: 1325-1326.

35. Cox DR. Regression Models and Life-Tables. J R Stat Soc Series B Stat Methodol. [Royal Statistical Society, Wiley]; 1972;34: 187-220.

36. Cha EDK, Xu J, Gong L, Gong H. Variations in Active Outflow Along the Trabecular Outflow Pathway. Exp Eye Res. Elsevier; 2016; doi:10.1016/j.exer.2016.01.008

37. Parikh HA, Loewen RT, Roy P, Schuman JS, Lathrop KL, Loewen NA. Differential Canalograms Detect Outflow Changes from Trabecular Micro-Bypass Stents and Ab Interno Trabeculectomy. Sci Rep. Nature Publishing Group; 2016;6: 34705.

38. Dang Y, Waxman S, Wang C, Parikh HA, Bussel II, Loewen RT, et al. Rapid learning curve assessment in an ex vivo training system for microincisional glaucoma surgery [Internet]. PeerJ Preprints; 2017 Jan. Report No.: e2745v1. doi:10.7287/peerj.preprints.2745v1

39. Loewen RT, Brown EN, Roy P, Schuman JS, Sigal IA, Loewen NA. Regionally Discrete Aqueous Humor Outflow Quantification Using Fluorescein Canalograms. PLoS One. 2016;11: e0151754.

40. Loewen RT, Brown EN, Scott G, Parikh H, Schuman JS, Loewen NA. Quantification of Focal Outflow Enhancement Using Differential Canalograms. Invest Ophthalmol Vis Sci. 2016;57: 2831-2838.

41. Xin C, Wang RK, Song S, Shen T, Wen J, Martin E, et al. Aqueous outflow regulation: Optical coherence tomography implicates pressure-dependent tissue motion. Exp Eye Res. 2016; doi:10.1016/j.exer.2016.06.007

42. Kim YG, Hong S, Lee CS, Kang SY, Seong GJ, Ma KT, et al. Level of vascular endothelial growth factor in aqueous humor and surgical results of ahmed glaucoma valve implantation in patients with neovascular glaucoma. J Glaucoma. journals.Iww.com; 2009;18: 443-447.

43. Esfandiari H, Pakravan . Mohammad, Loewen NA, Yaseri . Mehdi. The predictive value of early intraocular pressure and bleb morphology in mitomycin-C augmented trabeculectomy [Internet]. 
University of Pittsburgh; 2017. doi:10.13140/RG.2.2.12492.21122

44. Gedde SJ, Herndon LW, Brandt JD, Budenz DL, Feuer WJ, Schiffman JC, et al. Postoperative complications in the Tube Versus Trabeculectomy (TVT) study during five years of follow-up. Am J Ophthalmol. Bascom Palmer Eye Institute, Miller School of Medicine, University of Miami, Florida, USA. sgedde@med.miami.edu: 2012 Elsevier Inc; 2012;153: 804-814.e1.

45. Al-Torbak AA, Al-Shahwan S, Al-Jadaan I, Al-Hommadi A, Edward DP. Endophthalmitis associated with the Ahmed glaucoma valve implant. Br J Ophthalmol. 2005;89: 454-458.

46. Kaplowitz K, Chen X, Loewen N. Two year results for 180 degree trabectome ablation. San Francisco, CA: American Glaucoma Society Annual Meeting 2013. 2013.

47. Behndig A, Montan $P$, Stenevi U, Kugelberg M, Lundström M. One million cataract surgeries: Swedish National Cataract Register 1992-2009. J Cataract Refract Surg. 2011;37: 1539-1545. 


\section{Figures and Tables}

Figure 1

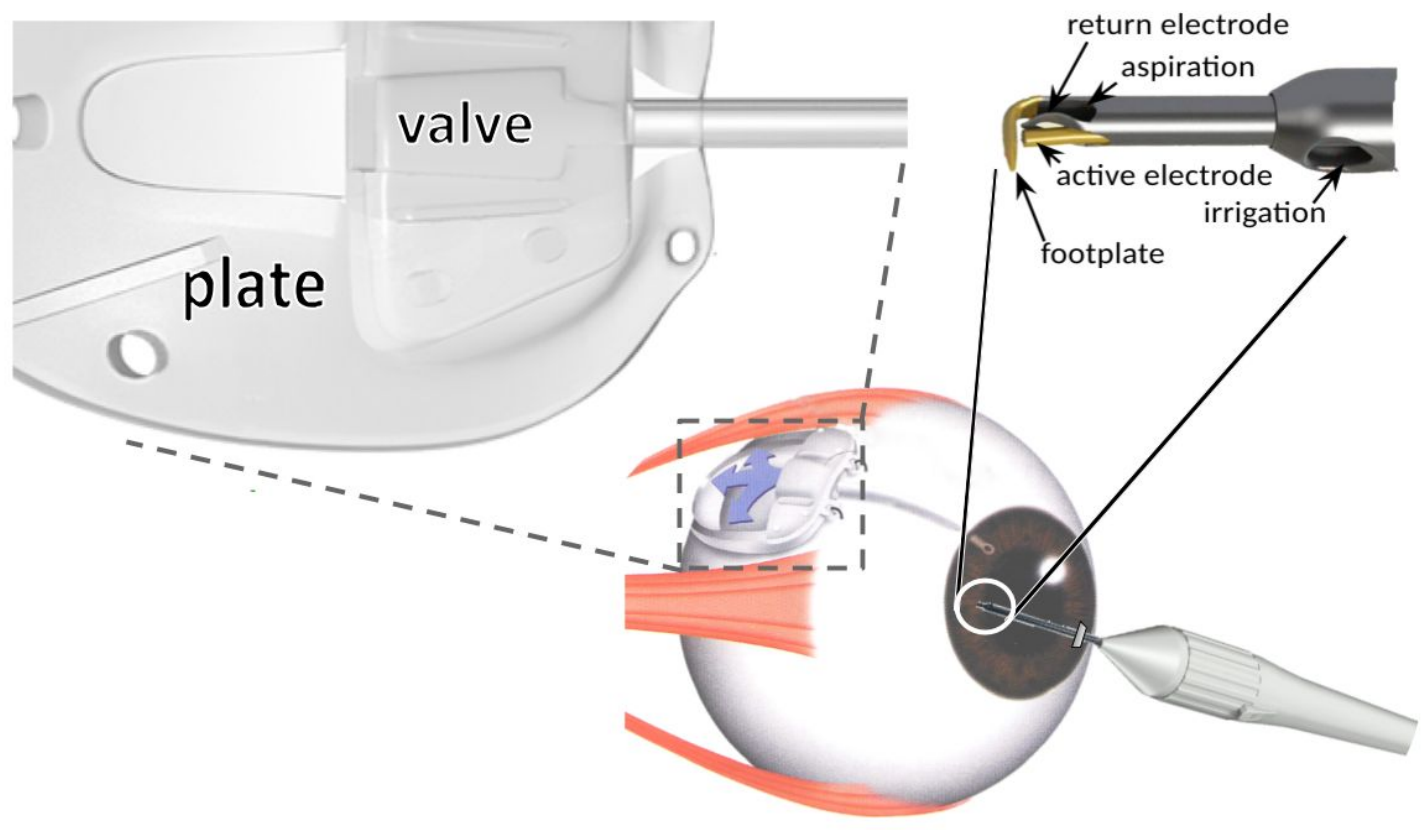

Fig 1. Ahmed glaucoma drainage device and Trabectome to scale. Devices are shown at the correct relative size and in the same eye for easier comparison. The Ahmed glaucoma drainage device has a valve to prevent overfiltration and a silicone plate with a surface area of $184 \mathrm{~mm}^{2}$ (left). The trabectome is most commonly inserted through a temporal incision (nasal incision depicted in this illustration), and the trabecular meshwork is ablated along the nasal angle. The tip of the trabectome has a protective footplate that feeds the trabecular meshwork towards the active and passive electrode where plasma is generated (top right). The molecularized tissue is displaced by fluid from the irrigation ports and aspirated at the tip. 
Figure 2

IOP in AGI and AIT

40

AGI
AIT

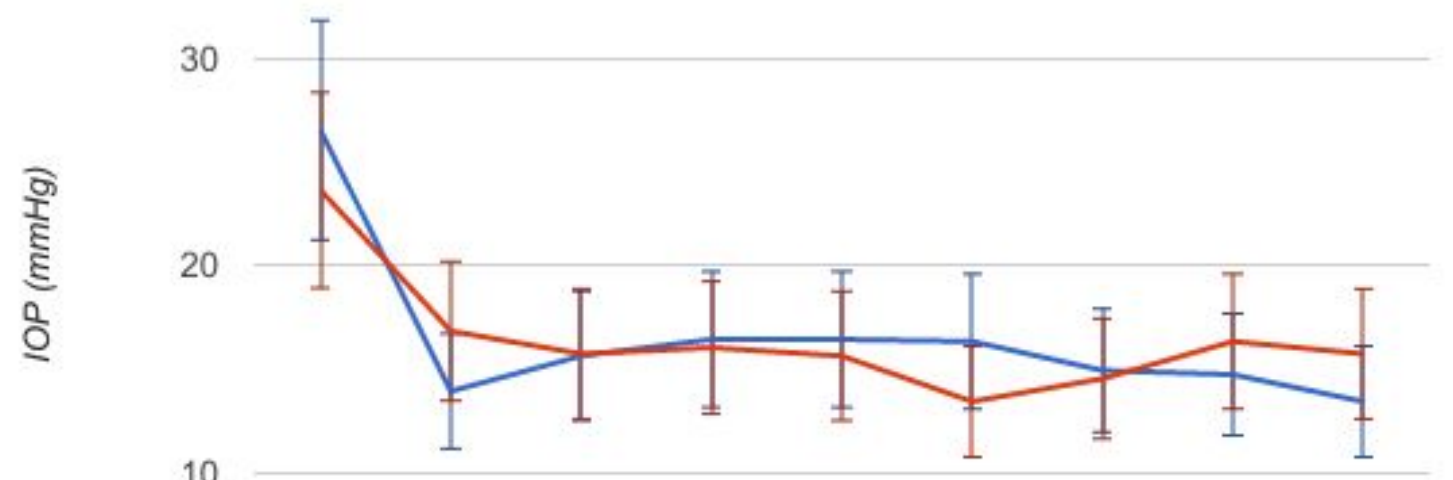

10

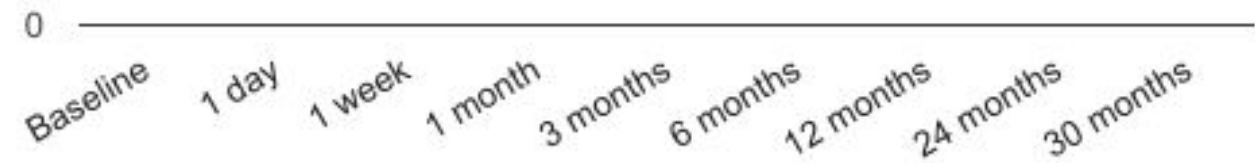

postoperative visit

Fig 2. Intraocular pressure after Trabectome-mediated ab interno trabeculectomy (AIT) and Ahmed glaucoma implant (AGI). The intraocular pressure (IOP, average \pm standard deviation) started diverging after three months but were not statistically different. Data for 24 months (AIT: $n=24, A G I: n=20$ ) and 30 months ( $A I T: n=18, A G I: n=12$ ) are also shown but were not part of the match due to reduced numbers. 
Figure 3

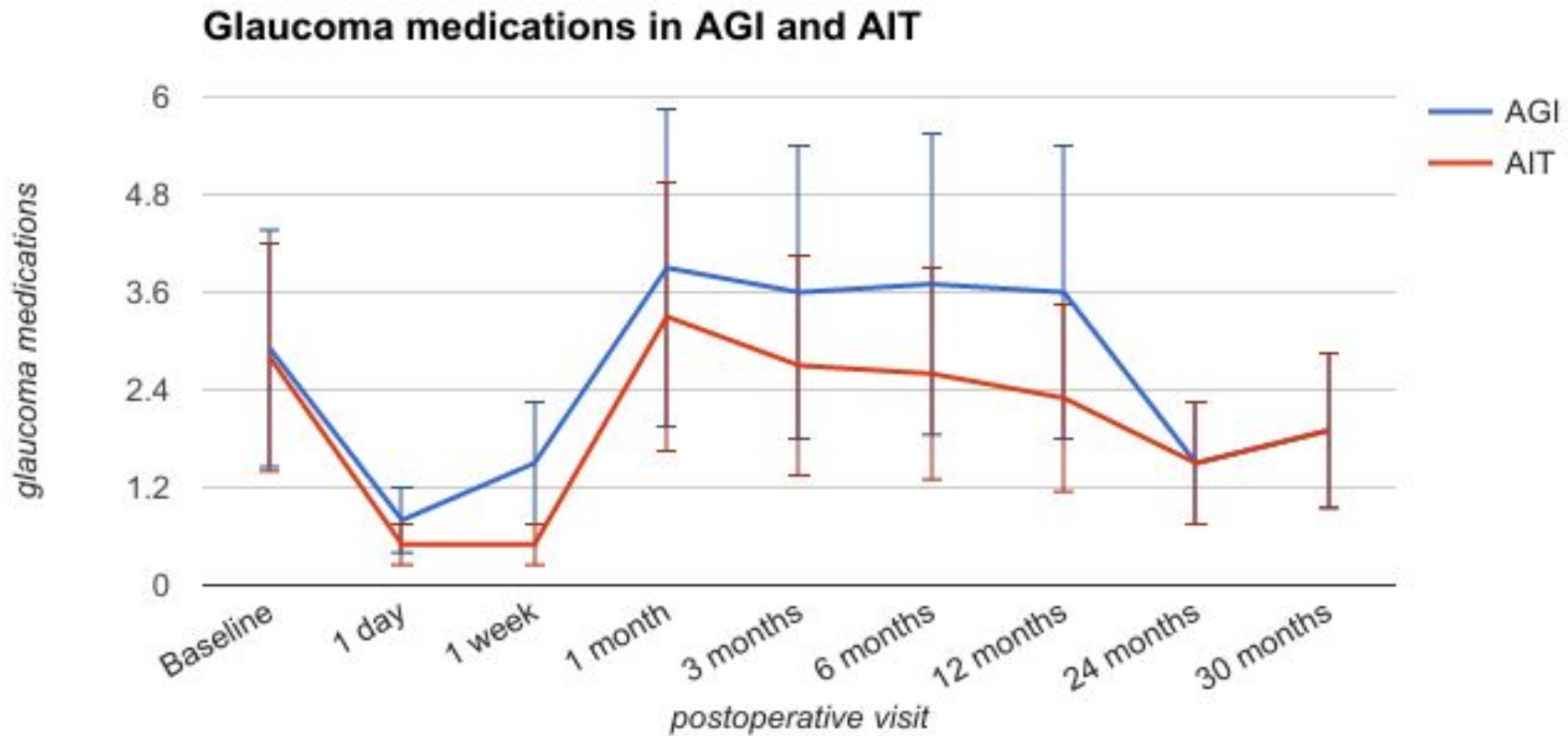

Fig 3. Glaucoma medications after Trabectome-mediated ab interno trabeculectomy (AIT) and Ahmed glaucoma implant (AGI). The number of glaucoma medications (average \pm standard deviation) started diverging after postoperative day 1 through 3 months but was not statistically different. Data for 24 months (AIT: $n=24, A G I: n=20$ ) and 30 months (AIT: $n=18, A G I: n=12$ ) are also shown but were not part of the match due to reduced numbers. 


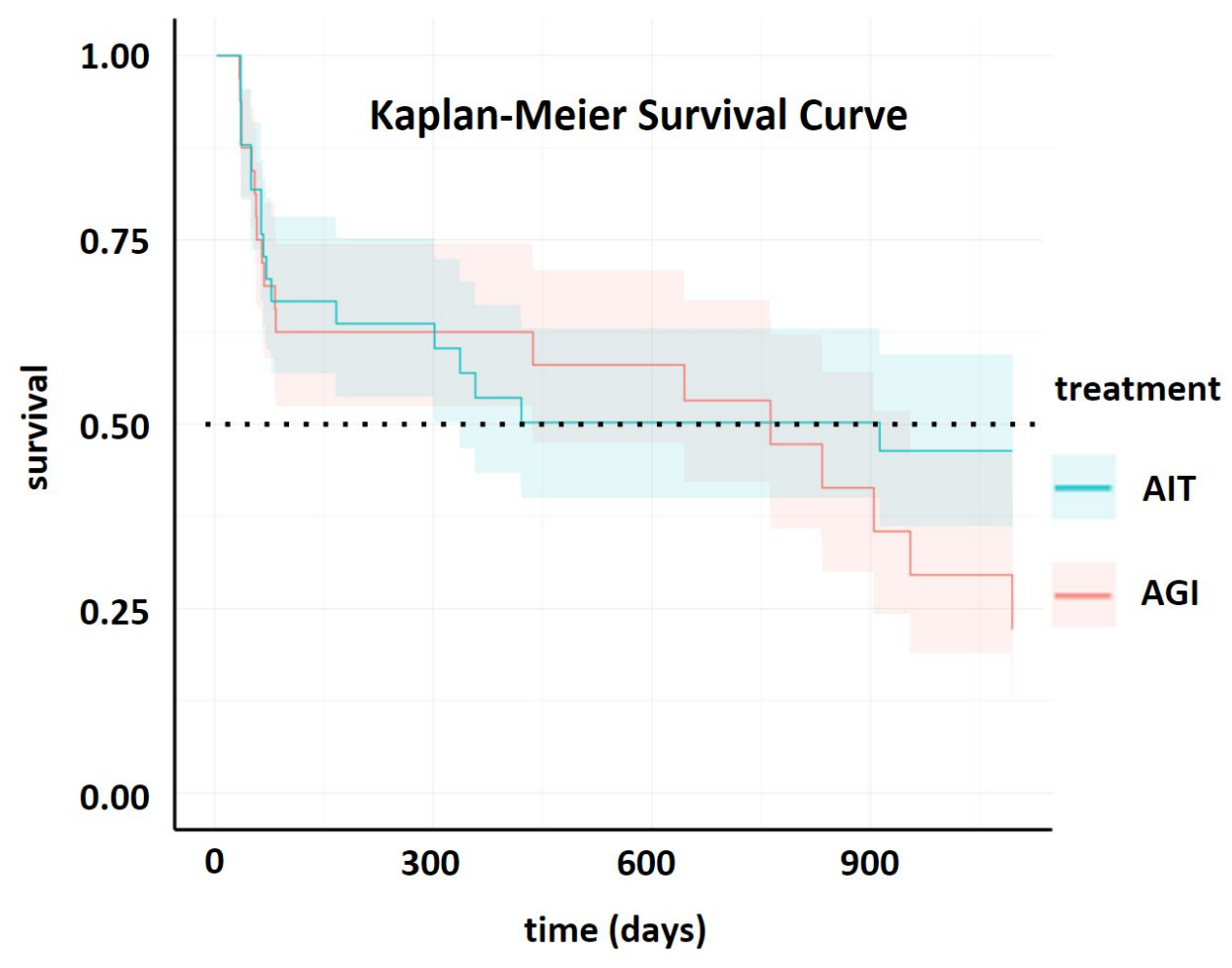

Fig 4. Survival analysis. Kaplan-Meier survival curve with $80 \%$ confidence intervals. AIT:

Trabectome-mediated ab interno trabeculectomy. AGI: Ahmed glaucoma drainage implant.
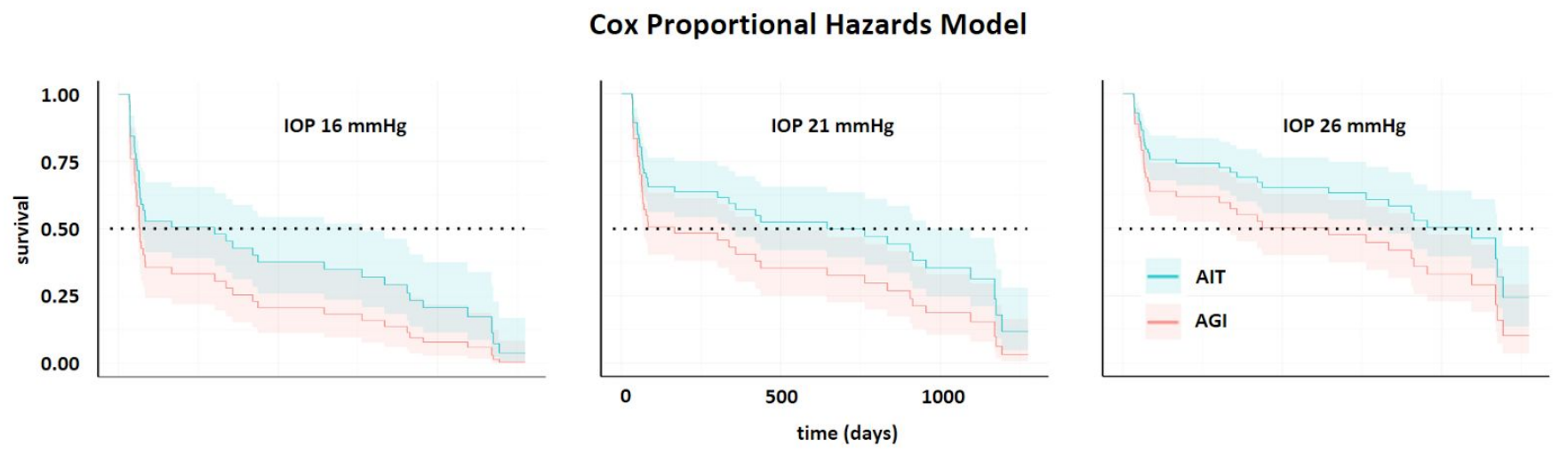

Fig 5. Cox hazard model. Kaplan-Meier survival curve (top left) and Cox proportional hazard models (right) with three different IOP criteria. The bands are the $80 \%$ confidence intervals. AGI: Ahmed 
glaucoma drainage implant. AIT: Trabectome-mediated ab interno trabeculectomy. 